\title{
Harvesting-Throughput Trade-off for Wireless-powered Smart Grid IoT Applications: An Experimental Study
}

\author{
Ecehan B. Pehlivanoglu* Mustafa Ozger* Oktay Cetinkaya* Ozgur B. Akan ${ }^{\dagger}$ \\ *Next-generation Wireless Communications Laboratory \\ Department of Electrical and Electronics Engineering \\ Koc University, 34450, Istanbul, Turkey \\ \{epehlivanoglu, mozger, okcetinkaya13\}@ku.edu.tr \\ $\dagger$ Internet of Everything (IoE) Group \\ Electrical Engineering Division, Department of Engineering \\ University of Cambridge, CB3 OFA Cambridge, UK \\ oba21@cam.ac.uk
}

\begin{abstract}
Sensor nodes, one of the most crucial elements of Internet of Things (IoT), sense the environment and send their observations to a remote Access Point (AP). One drawback of sensor nodes in an IoT setting is their limited battery supply. Hereby, energy harvesting (EH) stands as a promising solution to reduce or even completely eliminate lifetime constraints of sensors with exploitation of available resources. In this paper, we propose an electric-field EH (EFEH) method to enable batteryless execution of sensor-based IoT services for Smart Grid (SG) context. For this purpose, for the first time in the literature, harvestable energy through EFEH method is investigated with a transformer room experimental set-up. Our experiments reveal that $40 \mathrm{~mJ}$ of energy can be harvested in a period of 900 sec with the proposed EFEH method. Building on this energy profile, we define a throughput objective function $\theta$ for a "harvest-then-transmit" type system model, to shed light on the harvesting-throughput trade-off specific to IoT-assisted SG applications. Numerical results disclose non-trivial relationships between optimal harvesting period $T_{H}$, optimal transmission period $T_{T}$ and critical network parameters such as node-AP hop distance, path loss exponent and minimum reporting frequency requirement.
\end{abstract}

Index Terms-Electric-field, Energy Harvesting, Internet of Things, Smart Grid, Throughput Maximization.

\section{INTRODUCTION}

Internet of Things (IoT) provides a link between cyber and physical world by connecting devices over the Internet [1]. One of the key enabling technologies for IoT is Wireless Sensor Networks (WSNs), where wireless sensor nodes monitor the physical world, digitize their observations and send their readings to an Access Point (AP) that is connected to the Internet. Such a set-up enables sensor observations to be accessed remotely, whenever needed. However, operating wireless sensor nodes in an IoT setting with energy-limited batteries could be challenging as they are often deployed in large numbers and require frequent battery replenishments. In this context, energy harvesting $(\mathrm{EH})$ come into prominence to extend the lifetime of wireless devices by exploiting a stray source or converting useful energy from one form to another.

When an industrial facility in Smart Grid (SG) context is envisioned, continuity and reliability in operation are of utmost importance, as interruption in power provisioning cannot be tolerated in most cases. This necessitates real time monitoring of power systems over the Internet to better anticipate potential threats before they yield undesired consequences. Considering the harsh conditions in transformer rooms, which are the main pillars of SG applications, battery replenishments for wireless sensor nodes cannot be carried out without a system shut down, which in turn would interrupt power provisioning. Hence, EH approaches can be employed in SG context to altogether eliminate lifetime constraints of the sensors, evolving them to battery-less and self-operable entities, while ensuring reliable and interruption-free operation of SG pillars.

In simple terms, EH alleviates the limited lifetime problem of energy-constrained devices such as wireless sensor nodes [2]. The availability of conventional harvestable sources, namely light propagation, temperature gradient, motion variations and electromagnetic waves, are significantly affected by environmental variables or other external factors. Given this randomness, recent research interests on $\mathrm{EH}$ is focusing on investigating application specific solutions that are reliable, durable and profitable. In SG applications, alternatives of $\mathrm{EH}$ are highly limited for transformer room scenarios given the lack of ambient resources (e.g., light, air flow and/or temperature gradient) thereof. In addition, as there is neither noise nor motion variations in transformer rooms, electromagnetic (EM) fields in abundance are the only options for EH. When EM field-based techniques are envisioned for these settings, electric-field (E-field) oriented methods stand out compared to their magnetic-field (M-field) based counterparts, as they do not necessitate any galvanic contact with the transformer to harvest energy. To that end, E-field $\mathrm{EH}(\mathrm{EFEH})$ method is less complex, safer and less costly solution for battery-less operations of wireless devices in monitoring SG assets.

Preliminary works on EFEH were first performed on middle and high voltage transmission lines, exploiting the E-field in abundance [3]. The empirical findings revealed the efficiency of EFEH in providing advanced condition monitoring and remote control for SG architectures. EFEH has also been applied to low voltage systems as mounting commercial AC power 


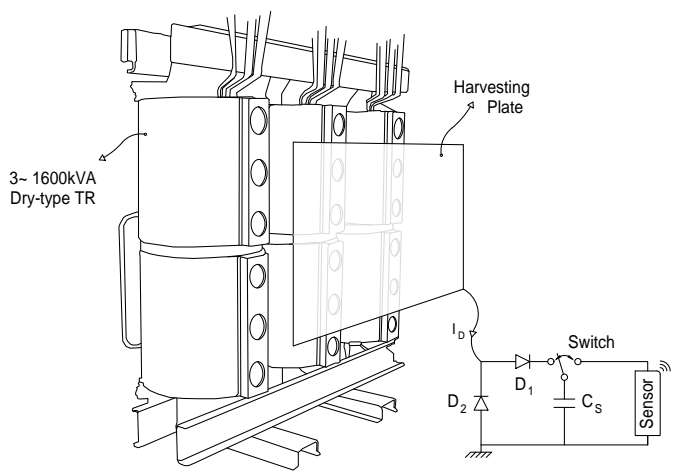

(a)

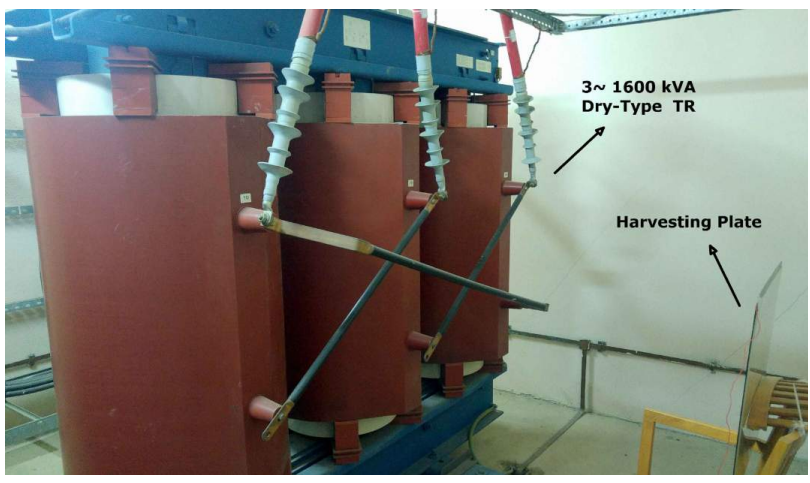

(b)

Fig. 1: (a) Representative diagram of the proposed EFEH method; (b) Experimental set-up of the proposed EFEH method.

cords with conductive metallic sheaths [4]. Results disclosed that it is possible to constitute an applicable procedure for applications in which the E-field intensity is considerably low. As an alternative to the previous studies, [5] has brought a new perspective with the proposed parallel plate model. This work introduced placement of a copper plate between troffers and ground plain to exploit the E-field flow around overhead fluorescent fixtures. The plate acts as a generic current source, and supports autonomous operation of wireless devices to be attached. The idea presented in [5] has been taken as the basis of the experimental set-up of this paper as well.

While studies that characterize the energy profile through EFEH are at early stages, many works focused on optimal utilization of the harvested energy by the the sensing and communicating entities. [6] is an excellent introduction, laying the cases for optimal use of the scavenged energy in terms of different utility functions such as maximum throughput or minimum outage probability. [7]-[13] are all great examples on optimal utilization of the extracted energy in wireless and cognitive radio ad hoc networks. However, none of these studies are based on neither an EFEH scheme nor an experimentbacked real energy profile. Additionally, these works consider that entirety of the exploited energy is consumed for communications; neglecting the energy consumed for nodal operations, such as waking up, sensing and processing. These operations actually account for a significant portion of the harvested energy according to our experimental results.

In this study, we investigate an EFEH method for a pointto-point communication architecture used in an IoT-assisted transformer room monitoring application. Through an experimental set-up, for the first time in the literature, we reveal the profile of harvestable energy in a transformer room with our proposed EFEH method. Building on this energy profile, in the second part, we theoretically study the harvesting-throughput trade-off for an EFEH wireless sensor node that is employed in a SG setting. The sensor node is assumed to periodically send its readings, in a single hop, to a distant AP that is connected to the Internet. To that end, the sensor node is following a "harvest-then-transmit" cycle; where it first harvests energy of amount $E_{H}$ during a period of $T_{H}$; then uses this energy for wake-up, sensing, processing and transmission operations.
The entire cycle is completed in a total duration of $T_{\text {Total }}$ and is periodically repeated. The aim is to ensure battery-less operation of the sensor node and to maximize the throughput function $\theta$ (i.e. the expected number of bits that are reliably conveyed to the AP in a single "harvest-then-transmit" cycle) while complying with a requirement on minimum frequency of reporting related to conditions of the transformer room. We reveal the changes in $\theta$ with respect to harvesting period $T_{H}$, transmission period $T_{T}$ and other critical system parameters.

The remainder of this paper is organized as follows. We first introduce the system model in Section II. Basic principles of EFEH are reviewed alongside a detailed description of the experimental set-up that we utilized for EFEH in Section II-A. Theoretical model on nodal operations and communication set-up employed in our work are described in Section II-B. In Section III, we define a throughput objective function $\theta$ to shed light on harvesting-throughput trade-off specific to IoT-assisted SG applications. In Section IV, we find the optimal harvesting period $T_{H}$ and transmission period $T_{T}$ which maximize throughput $\theta$, and investigate how they vary with respect to changes in critical network parameters. Finally, the paper is concluded in Section V.

\section{SYSTEM MODEL}

In this section, we first describe the experimental set-up that we utilized for EFEH in a transformer room, as a case study for IoT-assisted SG applications. System model details of the point-to-point communicating wireless sensor node that is powered with harvested energy are provided afterwards.

\section{A. Experimental Set-up for the Proposed EFEH Method}

According to electrostatics, electric charges distributed in a closed surface result in an E-field. In AC, this time varying field yields a displacement current, $I_{d}$, which can be defined with Maxwell's equation $I_{d}=\epsilon \int_{s} \frac{d \mathbf{E}}{d t} d s$, where $\epsilon$ is absolute permittivity, and $\mathbf{E}$ is the electric field intensity. The electric charges are dispatched and then collected in a storage element, $C_{s}$, via $I_{d}$. The energy stored in $C_{s}$ can be expressed as $E=$ $\frac{1}{2} C V_{s}^{2}$, where $C$ is capacitance of $C_{s}$ and $V_{s}$ is the voltage accumulated. As energy is gathered by exploiting the ambient field, this method can be referred to as Electric-field Energy Harvesting (EFEH) [3]-[5]. 

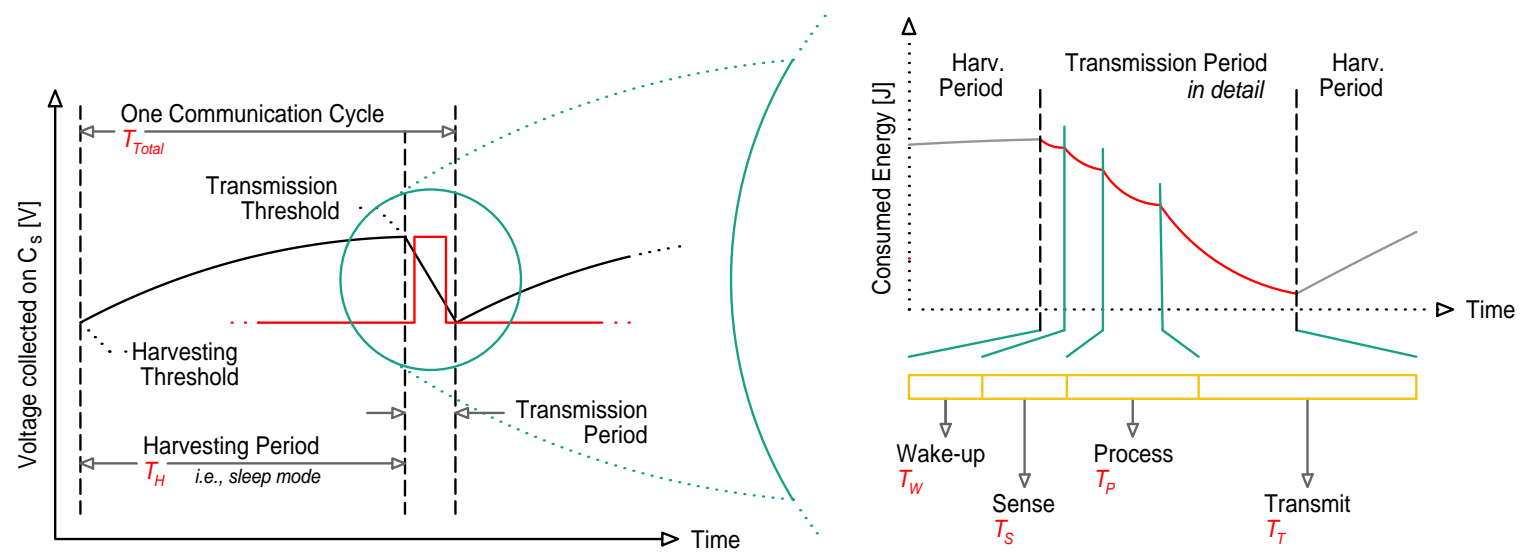

Fig. 2: Operation cycle of the proposed EFEH method.

Fig. 1(a) illustrates the diagram of the set-up that we built in a transformer room to harvest energy through EFEH method. A copper plate, i.e., the E-field energy harvester, is placed between the concrete wall and the primary side of a 3-phase $1600 \mathrm{kVA}$ dry-type transformer. The main objective of this plate is to collect the transformer (i.e. E-field) induced AC charges in $C_{s}$ by draining $I_{d}$, until the gathered voltage $V_{s}$ becomes adequate for sensor to perform wake up, sensing, processing, and data transmission processes. The diodes, $D_{1}$ and $D_{2}$, serve both to convert the harnessed energy from AC to DC, and to prevent it from backfeeding. The given 'Switch' model refers to an autonomous connection circuit to be employed for interchange between the operation stages, i.e., for the regulation of energy usage. This circuit allows charge conveyance when the accumulated energy $E_{H}$ is high enough for transmission, and switches off the sensory circuit when the voltage $V_{s}$ on $C_{s}$ drops below a certain threshold. This operation not only prevents $C_{s}$ from discharge down to $0 \mathrm{~V}$, but also enables better throughput by shortening the time spent on harvesting stage [4].

The actual implementation of this set-up is depicted in Fig. 1(b). As shown, a copper plate of $50 \times 50 \mathrm{~cm}^{2}$ size is used in the set-up as an E-field energy harvester. The location of the plate, or the safest possible distance for operation, is determined ideally to take advantage of capacitive coupling. As a result of high tension presented on the operation area, sharp corners of the plate are smoothened to avoid possible drawbacks related to corona-based partial discharges. Voltage and energy accumulated on $C_{s}$ with respect to time are revealed in detailed later in Section IV.

\section{B. Nodal Operation and Communication Model}

The wireless sensor node is assumed to be fed with the energy harvested through the proposed method of EFEH to carry out its nodal operations and communicate with an AP in a single hop, regarding the conditions of the transformer room and the transformer itself. This would enable notification of an upper level authority for decision-making procedures. By this means, preclusive actions can be performed against any unexpected events. With these actions, interruption-free operation of the SG medium can be guaranteed. The following set of facts and assumptions are taken into account regarding the operations of this node:

- The node is fed with the energy scavenged by the EFEH method, profile of which is determined through the experimental set-up described in Section II-A. It is assumed that there is no other external source of energy.

- The node's operations follow a cyclical pattern of duration $T_{T o t a l}$. Each cycle starts with a harvesting period, followed by a transmission period, as depicted in Fig. 2.

- The node operates in a half-duplex fashion given its hardware limitations, meaning it can only be in harvesting or transmission mode at any time [11].

- In each cycle, EFEH is carried out for a duration of $T_{H}$, accumulating a total energy of $E_{H}$.

- Transmission period consists of wake-up, sensing, processing and transmission operations, as in Fig. 2. These operations occupy durations of $T_{W}, T_{S}, T_{P}$ and $T_{T}$ in each cycle, respectively.

- The node is assumed to have enough data to send throughout the period $T_{T}$ in each cycle.

- Total duration of one cycle is $T_{\text {Total }}=T_{H}+T_{W}+T_{S}+$ $T_{P}+T_{T}$.

- The node is assumed to be "asleep" during the harvesting period, consuming a constant power $P_{Z}$ [14], resulting in an energy consumption of $E_{Z}=P_{Z} T_{H}$.

- The node is assumed to consume a constant $E_{N}=E_{W}+$ $E_{S}+E_{P}$ amount of energy for its nodal operations in each cycle.

- Due to energy causality constraint, consumed energy by the node within a cycle for wake-up, sensing, processing and transmission cannot exceed the energy available at that cycle after harvesting period is completed [8].

- The node follows an offline transmission policy, i.e., it has full knowledge of the amount and arrival time of harvested energy [7]. In fact, considering very low variability of the E-field in a transformer room, we assume the harvested energy profile does not change in time.

- The communication channel is assumed to be imperfect with Rayleigh fading. 


\section{HARVESTING-ThroughPUT TRADE-OFF}

Building on the system model presented in Section II, in this section we design one communication cycle of our EFEH-powered node. In each cycle, there are three orthogonal periods reserved for energy harvesting, nodal operations and transmission, as in Fig. 3. These periods serve the following:

- During the period $T_{H}$, the node exploits the E-field around the transformer, and subsequently charges $C_{s}$, reserving energy of amount $E_{H}$. Part of this reserve is already used during $T_{H}$, as the node consumes energy of amount $E_{Z}=P_{Z} T_{H}$ during sleep.

- $T_{N}=T_{W}+T_{S}+T_{P}$ is allocated for wake up, sensing and processing operations. Both the duration $T_{N}$ and energy consumption $E_{N}$ of this period are constants [14].

- In the last period of the cycle, the node has an energy budget of $E_{H}-E_{Z}-E_{N}$, which it needs to allocate in terms of transmission power $P_{T}$ and duration $T_{T}$, to send the maximum expected number of bits to the AP.

As part of the offline transmission policy, gathered energy $E_{H}$ in harvesting period $T_{H}$ is completely utilized within the same cycle, for energy consumptions in sleep mode during harvesting, nodal operations and transmission. Hence, we investigate one communication cycle of the node in operation, and look for optimal $T_{H}$ and $T_{T}$ values that would maximize the expected number of bits sent to the AP per second.

The harvesting period $T_{H}$ determines the energy captured in $C_{s}$. As $T_{H}$ is extended, scavenged energy $E_{H}$ increases non-linearly, with a decreasing rate as time progresses. There is an upper bound on the voltage $V_{s}$ that can be accumulated on $C_{s}$, which in turn limits the maximum amount of $E_{H}$. To that end, there is no logical reason to extend harvesting period beyond $T_{H_{\max }}$ when $V_{s}$ saturates, as seen in Fig. 3 . Initial energy reserve of $E_{H}$ is reduced to $E_{H}-E_{Z}-E_{N}$ by the time the transmission period is started. During this period, the node needs to find an optimal $T_{T}$ (hence $P_{T}$ ) to allocate this energy budget, maximizing the expected throughput.

In our optimization problem, the objective function is defined as the throughput of the communication channel between the wireless sensor node and the AP, denoted as $\theta$ :

$$
\theta=\frac{T_{T} B \log _{2}(1+\gamma)(1-B E R)^{\left(R_{b} T_{T}\right)}}{T_{\text {Total }}},
$$

where $T_{\text {Total }}=T_{H}+T_{N}+T_{T}, B E R$ is bit error rate, $B$ is the communication bandwidth, $\gamma$ is the received signal-tonoise ratio (SNR) and $R_{b}=B \log _{2}(1+\gamma)$ is the assumed channel bit rate. The optimization problem is formulated as:

$$
\begin{array}{cc}
\max _{T_{H}, T_{T}} & \frac{T_{T} B \log _{2}(1+\gamma)(1-B E R)^{\left(R_{b} T_{T}\right)}}{T_{\text {Total }}} \\
\text { s.t. } & T_{\text {Total }} \leq \frac{1}{f_{\text {min }}}, \\
& E_{H}>P_{Z} T_{H}+E_{N}
\end{array}
$$

The two constraints for this optimization problem are:

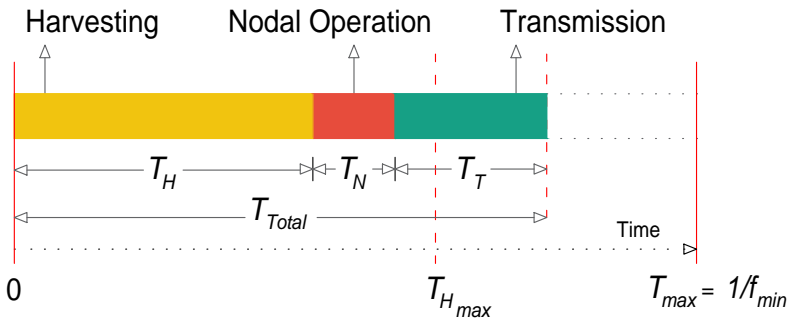

Fig. 3: Harvest-then-transmit cycle of the EFEH-powered node.

1) $T_{\text {Total }}$ must be less than or equal to inverse of minimum reporting frequency $f_{\min }$, which depends on requirements of the IoT-assisted SG monitoring application.

2) $T_{H}$ should be such that harvested energy $E_{H}$ would not be less than sum of the energy consumptions during sleep mode $\left(E_{Z}=P_{Z} T_{H}\right)$ and nodal operations $\left(E_{N}\right)$.

For the reliability of the packet, we first determine bit error rate (BER), which depends on SNR per bit, $\gamma_{b}$. The packet length is $R_{b} T_{T}$. Hence, the packet error probability is defined as $\left(1-B E R\left(\gamma_{b}\right)\right)^{R_{b} T_{T}}$, where $R_{b}=B \log _{2}(1+\gamma)$ is the bit rate of the channel and is assumed equal to the channel capacity. For Rayleigh fading channel, average BER for binary phase-shift keying (BPSK) is expressed as [15]

$$
\operatorname{BER}\left(\gamma_{b}\right)=\frac{1}{2}\left(1-\sqrt{\frac{\gamma_{b}}{1+\gamma_{b}}}\right) .
$$

SNR is defined as the ratio of the received signal power to the noise power, i.e., $\gamma=\frac{P_{R}}{N_{0} B}$. Accordingly, $\gamma_{b}=\frac{E_{b}}{N_{0}}$ refers to energy per bit over noise power density, i.e.,

$$
\frac{E_{b}}{N_{0}}=\frac{\gamma}{R_{b}}=\frac{P_{R}}{N_{0} B R_{b}},
$$

where $P_{R}$ is the power received by the access point [16]. $P_{R}$ is defined as

$$
P_{R}=\frac{P_{T} G_{t} G_{r} \lambda^{2}}{(4 \pi)^{2} L d^{\nu}},
$$

where $\lambda$ is the wavelength, $L$ is the receiver implementation loss, $d$ is the hop distance, $\nu$ is the path loss exponent, and $G_{t}$ and $G_{r}$ are the antenna gains of the transmitter and the receiver, respectively. The transmit power $P_{T}$ is provided from the residue of the harvested energy in one communication cycle. Hence, it is determined as $P_{T}=\frac{E_{H}-E_{Z}-E_{N}}{T_{T}}$.

Throughput expression, $\theta$, corresponds to the expected number of bits that are correctly transmitted to the AP over one communication period. We find $T_{H}$ and $T_{T}$ values that would maximize $\theta$ for different parameters such as hop distance $(d)$, path loss exponent $(v)$ and minimum reporting frequency $\left(f_{\min }\right)$ in Section IV. We denote the optimal harvesting and transmission periods as $T_{H}^{*}$ and $T_{T}^{*}$, respectively.

\section{Numerical Results}

In this section, we study the optimal harvesting and transmission periods, $T_{H}^{*}$ and $T_{T}^{*}$, that would maximize the objective function $\theta$, which corresponds to the throughput in between the sensor node-AP system in one communication cycle. The constants that are used for the numerical study of the $\theta$ function are given in Table I. 


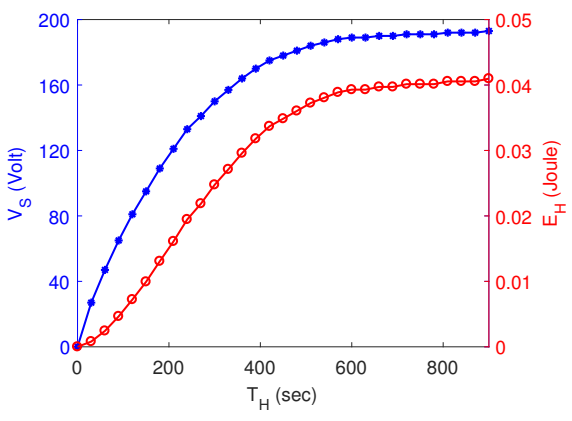

(a)

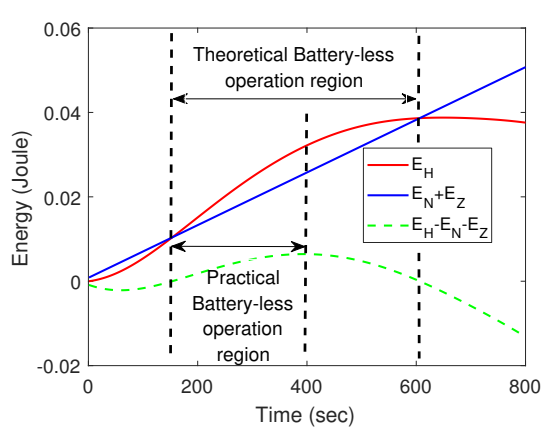

(b)

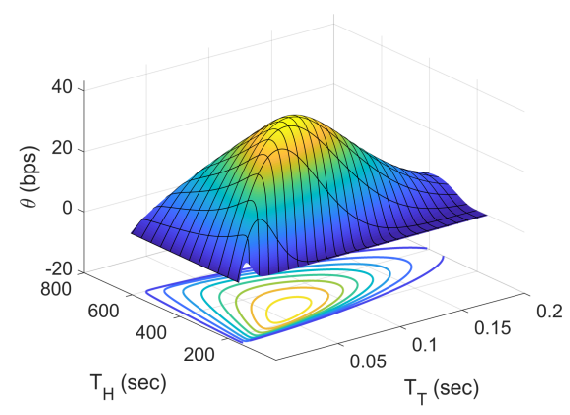

(c)

Fig. 4: (a) Accumulated voltage $V_{s}$ and energy $E_{H}$ on capacitor $C$ with respect to $T_{H}$; (b) Accumulated energy $E_{H}$ and consumed energy for sleep mode and nodal operations $E_{N}+E_{Z}$ with respect to $T_{H}$; (c) Value of objective function $\theta$ with respect to $T_{H}$ and $T_{T}$.

TABLE I: Constant parameters and their values to evaluate $\theta$ [14]

\begin{tabular}{||c|c||}
\hline Parameter & Value \\
\hline \hline$P_{Z}$ & $6.2 \times 10^{-5} \mathrm{~W}$ \\
\hline$E_{W}$ & $1.6 \times 10^{-5} \mathrm{~J}$ \\
\hline$E_{S}$ & $6.4 \times 10^{-4} \mathrm{~J}$ \\
\hline$E_{P}$ & $1.6 \times 10^{-4} \mathrm{~J}$ \\
\hline$T_{W}$ & $10^{-2} \mathrm{sec}$ \\
\hline$T_{S}$ & $6.7 \times 10^{-2} \mathrm{sec}$ \\
\hline$T_{P}$ & $2.2 \times 10^{-3} \mathrm{sec}$ \\
\hline$B$ & $10 \mathrm{kHz}$ \\
\hline$N_{0}$ & $4.17 \times 10^{-21} \mathrm{~W} / \mathrm{Hz}$ \\
\hline$\lambda$ & $0.125 \mathrm{~m}$ \\
\hline$G_{t}, G_{r}, L$ & 1 \\
\hline
\end{tabular}

As the first step, we reveal the harvested energy $E_{H}$ with respect to harvesting period $T_{H}$, according to experimental results from the EFEH set-up illustrated earlier in Fig. 1(a). Based on measurements from the actual transformer room visualized in Fig. 1(b), for a capacitance of $2.2 \mu \mathrm{F}$, the accumulated voltage $V_{s}$ on $C_{s}$ increases up to $195 \mathrm{~V}$ and saturates when the harvesting time reaches to $900 \mathrm{sec}$ as seen in Fig. 4(a). In a similar fashion, the accumulated energy $E_{H}$ saturates at $40 \mathrm{~mJ}$ in the same time period. The shapes of $V_{s^{-}}$ $T_{H}$ and $E_{H}-T_{H}$ curves can be altered by employing different capacitors in the set-up, and accumulated energy at saturation would be higher for a bigger capacitance, assuming the voltage induced by the EFEH source (which acts as a non-ideal current source) does not exceed the safe voltage limit of the capacitor.

The $E_{H}-T_{H}$ curve is specifically important not only because it dictates the region(s) where the sensor node-AP system can operate in a battery-less manner, but also influences where this system would have the optimal throughput as defined by the $\theta$ function. For battery-less operation, $E_{H}$ should be higher than the energy consumed for sleep mode during harvesting, $E_{Z}$, and the energy consumed for subsequent nodal operations of wake up, sensing and processing, i.e., $E_{N}=E_{W}+E_{S}+E_{P}$. As illustrated in Fig. 4(b), this is achieved when $T_{H}$ lies in the $154-605 \mathrm{sec}$ interval. This is the theoretical battery-less operation region of our system. However, as depicted by the dashed line in Fig. 4(b), there is no logical reason to extend $T_{H}$ in a cycle beyond $390 \mathrm{sec}$, as the reserve energy $E_{H}-E_{N}-E_{Z}$ starts to decrease at that point. Hence, 154-390 sec interval is the practical battery-less region of our system. In summary, any transformer room monitoring application that requires a reporting frequency of more than once in $154 \mathrm{sec}$ cannot be realized with the EFEH set-up in consideration. Additionally, if the application's reporting frequency requirement is less than once in $390 \mathrm{sec}$, the system is still better off from a throughput perspective to do reporting sometime in the interval of 154$390 \mathrm{sec}$ in each cycle.

As the second step, building on the identified $E_{H}-T_{H}$ curve and the battery-less operation regions, we examine the variation of throughput function $\theta$ with respect to $T_{H}$ and $T_{T}$. With the distance between the wireless sensor node and AP set to $d=5 \mathrm{~m}$, a path loss exponent of $\nu=2.5$ and a minimum reporting frequency of $f_{\min }=\frac{1}{800}$; the $\theta$ function with respect to $T_{H}$ and $T_{T}$ is depicted in Fig. 4(c). Sections of $\theta$ for different $T_{H}$ and $T_{T}$ values are concave functions, and $\theta$ has one global maximum. For this case, optimal values that maximize $\theta$ are $T_{H}^{*}=266.5 \mathrm{sec}$ and $T_{T}^{*}=60.5$ msec. Consequently, $\theta=43.24$ bps and in a duration of $T_{\text {Total }}=T_{H}+T_{N}+T_{T}=266.64 \mathrm{sec}$, sensor node can share 11.53 kbits with the AP in this optimal setting.

As the third step, we study how $T_{H}^{*}$ and $T_{T}^{*}$ values that maximize $\theta$ function vary with respect to distance $d$ between the sensor node-AP system and the path loss exponent $\nu$ of the channel. Fig. 5 reveals these optimal values for (a) $\nu=2$, (b) $\nu=2.5$ and (c) $\nu=3$; where in each case $d$ is swept from $3 \mathrm{~m}$ to $250 \mathrm{~m}$. The first and foremost result is that $T_{H}^{*}$ is very robust to changes in $d$ and $\nu$. In fact, $T_{H}^{*}$ varies very slightly from $276 \mathrm{sec}$ to $277 \mathrm{sec}$, as $d$ is increased from $3 \mathrm{~m}$

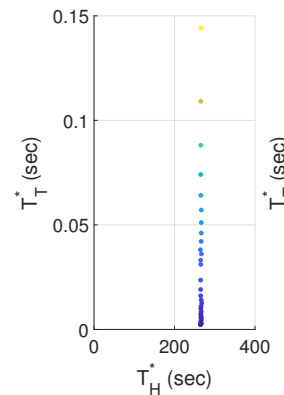

(a)

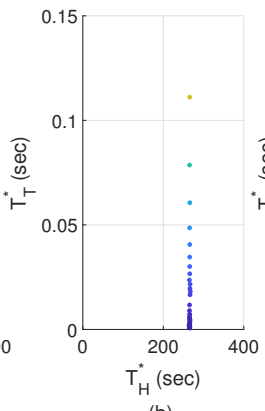

(b)

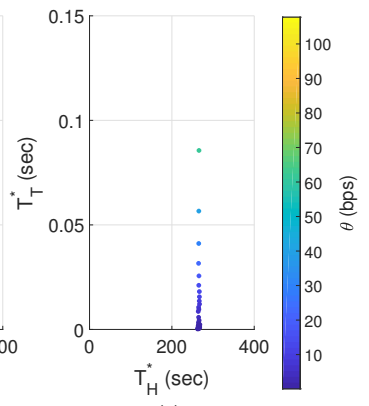

(c)
Fig. 5: $T_{H}^{*}$ and $T_{T}^{*}$ values that maximize $\theta$, for path loss exponents of (a) $\nu=2$, (b) $\nu=2.5$ and (c) $\nu=3$, respectively. 


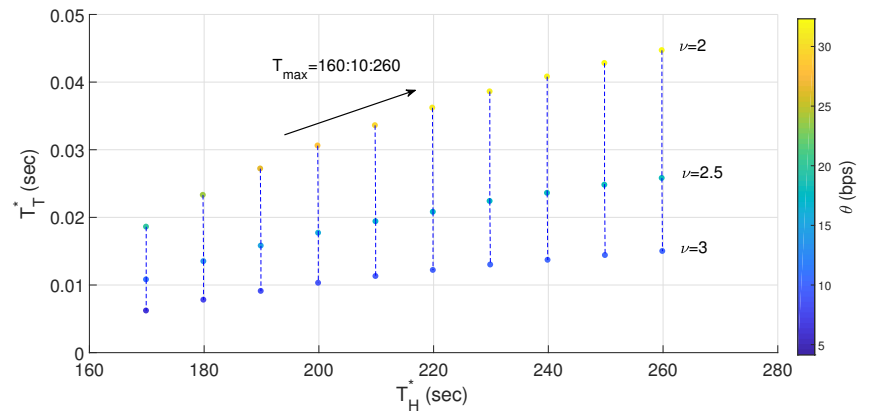

Fig. 6: $T_{H}^{*}$ and $T_{T}^{*}$ values that maximize $\theta$, for path loss exponents of $\nu=2: 0.5: 3$, where $T_{\max }$ is swept from 160 to $300 \mathrm{sec}$. Points with same $T_{\max }$ are connected with dashed lines.

to $250 \mathrm{~m}$ and $\nu$ is increased from 2 to 3 . This stems from the fact that denominator of $\theta$ in Eq. (2) is dominated by $T_{H}$, as $T_{H}>T_{T}$, given the energy harvesting speed of the proposed EFEH method. Consequently, $\theta$ is maximized in close proximity of $T_{H}=266 \mathrm{sec}$, where the $E_{H}-T_{H}$ curve turns from concave upward to concave downward. At this exact point, reserve energy $E_{H}-E_{N}-E_{Z}$ available for transmission in ratio to $T_{H}$ is maximized. In short, energy profile $E_{H}-T_{H}$ is the dominant driver of $T_{H}^{*}$, and it can be altered by varying the capacitance in the circuitry of Fig. 1(a). A different capacitance would change the $T_{H}^{*}$, as it represents the inflection point of the $E_{H}-T_{H}$ curve. On the other hand, $T_{T}^{*}$ is very sensitive to changes in $d$ and $\nu$, as seen in Fig. 5 . Taking $\nu=2.5$ case as an example, it varies from $T_{T}^{*}=111$ msec for $d=3 \mathrm{~m}$ to $T_{T}^{*}=16.5 \mathrm{msec}$ for $d=250 \mathrm{~m}$. As conditions get harsher with a longer distance and a higher path loss exponent, $T_{T}^{*}$ is contracted to obtain relatively high SNR values in order to keep the BER manageable.

As the last step, effect of minimum reporting frequency $f_{\min }$ on $T_{H}^{*}$ and $T_{T}^{*}$ is investigated. This $f_{\min }$ depends on the variable that is being monitored by the sensor node and translates into an upper limit on $T_{\text {Total }}$ as $T_{\text {Total }}=T_{H}+T_{N}+$ $T_{T} \leq \frac{1}{f_{\min }}$. As seen already in Fig. 4(b), any application requirement that implies $\frac{1}{f_{\min }}=T_{\max }<154 \mathrm{sec}$ cannot be fulfilled with the proposed system model. For $d=10 \mathrm{~m}$, Fig. 6 illustrates how $T_{H}^{*}$ and $T_{T}^{*}$ varies with $T_{\max }$ and $\nu$. As an example, without a restricting requirement on reporting frequency, $T_{H}^{*}=266.3 \mathrm{sec}$ and $T_{T}^{*}=46 \mathrm{msec}$ for $d=10$ and $\nu=2$. Under same channel conditions, with $f_{\min }=\frac{1}{200}$ requirement set, these values shift to $T_{H}^{*}=199.89 \mathrm{sec}$ and $T_{T}^{*}=30.6 \mathrm{msec}$. In a sense, a restricting requirement on $f_{\text {min }}$ forces the system to end up in a sub-optimal setting in terms of $\theta$. That being said, loss in $\theta$ compared to its global maximum due to this sub-optimality is less than $20 \%$ for all $f_{\min }<\frac{1}{190}$. Additionally, as $f_{\min }$ requirement is gradually relaxed, the trajectory of the $T_{T}^{*}$ is concave downward, with diminishing returns on $\theta$. As an example, $\theta$ increases almost by a factor $2 \times$ when $f_{\min }$ is relaxed from $\frac{1}{160}$ to $\frac{1}{200}$, whereas it increases by a mere $10 \%$ as it is further extended to $\frac{1}{300}$.

\section{CONCLUSiON}

In this paper, we propose an EFEH method to enable battery-less execution of sensor-based IoT services for $\mathrm{SG}$ context. For the first time in the literature, harvestable energy through EFEH is investigated with a transformer room experimental set-up. Our experiments reveal that $40 \mathrm{~mJ}$ of energy can be harvested in a period of $900 \mathrm{sec}$ with the proposed EFEH method. Afterwards, we define a throughput objective function $\theta$ for a "harvest-then-transmit" type system model, to shed light on the harvesting-throughput trade-off specific to IoTassisted SG applications. Accordingly, battery-less execution of this system is plausible when harvesting period $T_{H}$ lies in the interval of $154-390 \mathrm{sec} . T_{H}^{*}$ is very robust to changes in conditions such as hop distance $d$ and path loss exponent $\nu$. That being said, $T_{H}^{*}$ is mainly driven by the energy profile $E_{H^{-}}$ $T_{H}$, which can be altered by varying the capacitance in the circuitry of the proposed EFEH method. Having a requirement on $f_{\text {min }}$ forces the system to end up in a sub-optimal setting in terms of $\theta$. However, loss in $\theta$ compared to its global maximum is not significant unless it pushes $T_{\max }$ very close to lower end of the battery-less operation region.

\section{REFERENCES}

[1] L. Atzori, A. Iera, G. Morabito, "The Internet of Things: A survey," Computer Networks, vol. 54, no. 15, pp. 2787-2805, Oct. 2010.

[2] S. Sudevalayam, and P. Kulkarni, "Energy harvesting sensor nodes: Survey and implications," IEEE Comm. Surveys \& Tutorials, vol. 13, no. 3, pp. 443-461, Sept. 2011.

[3] R. Moghe, Y. Yang, F. Lambert, and D. Divan, "A scoping study of electric and magnetic field energy harvesting for wireless sensor networks in power system applications," Proc. IEEE Energy Conv. Congr. \& Expo., Sept. 2009, pp. 3550-3557.

[4] O. Cetinkaya, O. B. Akan, "Electric-field Energy Harvesting Wireless Networks," IEEE Wireless Communications, vol. 24, no. 2, pp. 34-41, Apr. 2017.

[5] O. Cetinkaya and O. B. Akan, "Electric-field Energy Harvesting from Lighting Elements for Battery-less Internet of Things, IEEE Access, vol. 5, pp. 7423-7434, Apr. 2017.

[6] H. Li, J. Xu, R. Zhang and S. Cui, "A general utility optimization framework for energy-harvesting-based wireless communications," IEEE Communications Magazine, vol. 53, no. 4, pp. 79-85, April 2015.

[7] Y. He, X. Cheng, W. Peng and G. L. Stuber, "A survey of energy harvesting communications: models and offline optimal policies," IEEE Communications Magazine, vol. 53, no. 6, pp. 79-85, June 2015.

[8] O. Ozel, K. Tutuncuoglu, S. Ulukus and A. Yener, "Fundamental limits of energy harvesting communications," IEEE Communications Magazine, vol. 53, no. 4, pp. 126-132, April 2015.

[9] A. Sultan, "Sensing and Transmit Energy Optimization for an Energy Harvesting Cognitive Radio," IEEE Wireless Communications Letters, vol. 1, no. 5, pp. 500-503, October 2012.

[10] S. Yin, E. Zhang, L. Yin and S. Li, "Optimal saving-sensing-transmitting structure in self-powered cognitive radio systems with wireless energy harvesting," IEEE ICC, pp. 2807-2811, 2013.

[11] M. Zheng, C. Xu, W. Liang and H. Yu, "Harvesting-throughput tradeoff for RF-powered underlay cognitive radio networks," Electronics Letters, vol. 52, no. 10, pp. 881-883, December 2016.

[12] S. Yin, E. Zhang, L. Yin and S. Li, "Saving-sensing-throughput tradeoff in cognitive radio systems with wireless energy harvesting," IEEE Global Communications Conference (GLOBECOM), pp. 1032-1037, 2013.

[13] M. Zheng, W. Liang and H. Yu, "Harvesting-Throughput Tradeoff for CDMA-Based Underlay Cognitive Radio Networks With Wireless Energy Harvesting," IEEE Systems Journal, vol. PP, no. 99, pp. 1-4, 2016.

[14] S. Chamanian et al., "Powering-up wireless sensor nodes utilizing rechargeable batteries and an electromagnetic vibration energy harvesting system," Energies vol. 7, no. 10, pp. 6323-6339, Oct. 2014.

[15] A. Goldsmith, Wireless Communications. Cambridge, U.K.: Cambridge Univ. Press, 2005.

[16] M. C. Oto and O. B. Akan, "Energy-Efficient Packet Size Optimization for Cognitive Radio Sensor Networks," IEEE Transactions on Wireless Communications, vol. 11, no. 4, pp. 1544-1553, 2012. 